\title{
Time to diagnosis and mortality in colorectal cancer: a cohort study in primary care
}

\author{
ML Tørring ${ }^{*, 1,2}$, M Frydenberg ${ }^{3}$, RP Hansen', F Olesen', W Hamilton ${ }^{4}$ and P Vedsted' \\ 'The Research Unit for General Practice, Research Centre for Cancer Diagnosis in Primary Care, School of Public Health, Aarhus University, Bartholin Allé 2, \\ DK-8000 Aarhus C, Denmark; ${ }^{2}$ Section for General Medical Practice, School of Public Health, Aarhus University, Bartholin Allé 2, DK-8000 Aarhus C, \\ Denmark; ${ }^{3}$ Department of Biostatistics, School of Public Health, Aarhus University, Bartholin Allé 2, DK-8000 Aarhus C, Denmark; ${ }^{4} \mathrm{NIHR}$ School for \\ Primary Care Research, Department of Community Based Medicine, University of Bristol, 25/27 Belgrave Road, Bristol, BS8 2AA, UK
}

BACKGROUND: The relationship between the diagnostic interval and mortality from colorectal cancer (CRC) is unclear. This association was examined by taking account of important confounding factors at the time of first presentation of symptoms in primary care. METHODS: A total of 268 patients with CRC were included in a prospective, population-based study in a Danish county. The diagnostic interval was defined as the time from first presentation of symptoms until diagnosis. We analysed patients separately according to the general practitioner's interpretation of symptoms. Logistic regression was used to estimate 3-year mortality odds ratios as a function of the diagnostic interval using restricted cubic splines and adjusting for tumour site, comorbidity, age, and sex.

RESULTS: In patients presenting with symptoms suggestive of cancer or any other serious illness, the risk of dying within 3 years decreased with diagnostic intervals up to 5 weeks and then increased $(P=0.002)$. In patients presenting with vague symptoms, the association was reverse, although not statistically significant.

CONCLUSION: Detecting cancer in primary care is two sided: aimed at expediting ill patients while preventing healthy people from going to hospital. This likely explains the counterintuitive findings; but it does not explain the increasing mortality with longer diagnostic intervals. Thus, this study provides evidence for the hypothesis that the length of the diagnostic interval affects mortality in CRC patients.

British Journal of Cancer (201 I) 104,934-940. doi:10.1038/bjc.2011.60 www.bjcancer.com

Published online I March 201 |

(c) 201। Cancer Research UK

Keywords: primary health care; colorectal cancer; delayed diagnosis; waiting lists; mortality

Studies have shown large variations in the diagnostic intervals (the time from the first presentation of symptoms in primary care until diagnosis) for colorectal cancer (CRC) patients, with some patients experiencing intervals of months (Barrett et al, 2006; Korsgaard et al, 2006; Hansen et al, 2008; Terhaar et al, 2010). Although early diagnosis of cancer is generally deemed desirable, some clinicians and researchers are sceptic about the benefits of expediting diagnosis for CRC patients (Irvin and Greaney, 1977; Bako et al, 1988; Rupassara et al, 2006). The reasoning is that diagnostic intervals, measured in weeks to months, may be negligible by comparison with the time required for the development of the tumour, measured in years (Polissar et al, 1981; Pescatori et al, 1982; Auvinen, 1992; Iversen et al, 2009; Terhaar et al, 2010).

Studies have shown an important, but modest, reduction in CRC mortality by screening, indicating that time to diagnosis may matter (Hewitson et al, 2007; Atkin et al, 2010). However, the majority of CRC patients are diagnosed after presenting with symptoms, and no randomised trials have examined the benefits of expediting diagnosis in symptomatic patients (Mitchell et al, 2008). Most of the published literature report data, inevitably, from observational studies. A recent review indicated a lack of

*Correspondence: ML Tørring; E-mail: mlt@alm.au.dk

Received 8 November 2010; revised 23 January 2011; accepted 26 January 20 I I; published online I March 20I I association between the diagnostic interval and mortality in CRC patients, but the authors emphasised that future studies should focus on adjusting for confounding factors, as many of the included studies had counterintuitive results, showing that patients with short diagnostic intervals had higher mortality than patients with long diagnostic intervals (Ramos et al, 2007). Such findings are usually referred to as the 'waiting time paradox' and have also been reported for other types of cancer (Neal, 2009).

The aim of this study was to analyse the association between the diagnostic interval and mortality after diagnosis of CRC, while taking account of the interpretation of symptoms by the general practitioner (GP) and controlling for confounding factors at first presentation.

\section{MATERIALS AND METHODS}

We conducted a cohort study with overall 3-year mortality as the primary outcome and the diagnostic interval as the exposure variable.

\section{Setting}

The cohort resided in the former Aarhus County, Denmark, which has 640000 inhabitants and $\sim 400$ new CRC cases per year (NORDCAN, 2009). Denmark's publicly funded health-care system provides free access to general practice and hospital care. More 
than $98 \%$ of Danish citizens are registered with a GP whom they have to consult for medical advice. The GP acts as a gatekeeper to the rest of the health-care system, carrying out initial diagnostic investigations and referring patients to hospitals or outpatient clinics when necessary. Danish GPs are legally bound to keep detailed medical records of their patients (Christiansen, 2002).

\section{Study population}

Our study included all newly diagnosed CRC patients over the age of 17 during 1 year (1 September 2004 to 31 August 2005). Subsequently, the study population was restricted to the $89.8 \%$ of patients who had attended general practice before the cancer diagnosis (see flowchart, Figure 1).

\section{Identification of patients and GPs}

Register data for this study were linked by means of the civil registry number, a unique personal identifier assigned to all Danish citizens at birth. Patients were identified from the County Hospital Discharge Registry, a population-based medical database that records dates of all inpatient and outpatient visits and discharge diagnoses classified according to the International Classification of Diseases (ICD-10). We defined incident CRC patients as patients registered with a first-time discharge diagnosis of colon cancer (C18 and C19) or rectal cancer (C20) during the study period; and with no previous diagnosis of colon cancer, rectal cancer, or malignant neoplasm of ill-defined, secondary, and unspecified sites (C76-80).

In 2009, we verified all diagnoses by comparing the data with the Danish Cancer Registry, which records all incident cancer cases in Denmark and holds key information from the Danish National

IDENTIFIED patients with a first-time discharge diagnosis of CRC in the County Hospital Discharge Registry: 393

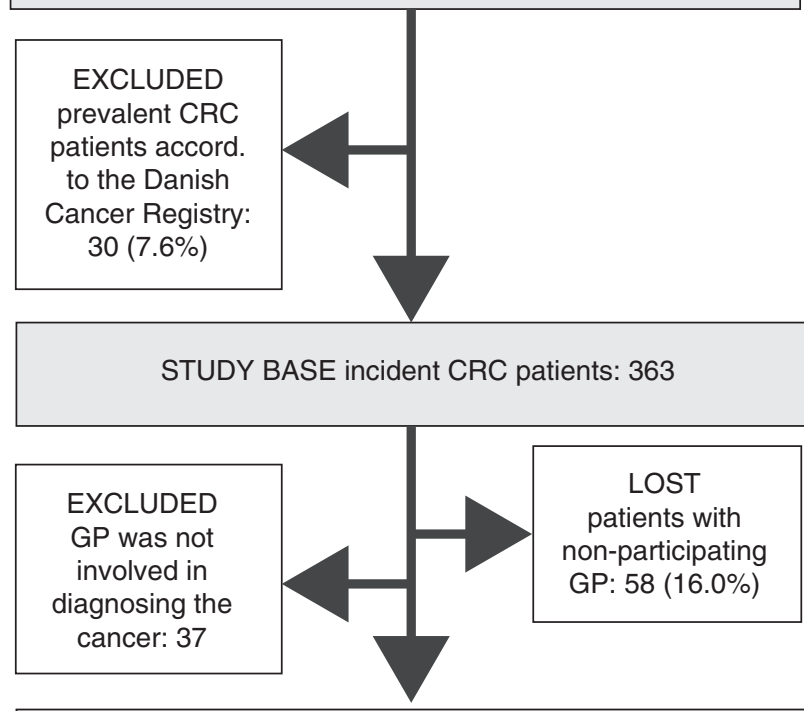

STUDY SUBJECTS incident cancer patients attending primary care before diagnosis: 268

Figure I Flowchart showing identification of incident colorectal cancer (CRC) patients in Aarhus County, Denmark, 2004-2005, for whom general practice was involved in diagnosing the cancer. The last criterion could not be determined for patients with nonparticipating GPs.
Pathology Registry. To identify the patients' GPs, the data were linked to the County Health Service Registry (Sørensen, 2009).

\section{Data collection}

A questionnaire was sent to each patient's GP. In practices with more than one GP, we asked the GP most familiar with the patient to complete the questionnaire. The GPs received compensation for their participation (DKK 240 equivalent to EUR 32). Nonresponders received a reminder after 3 weeks. The GPs were asked to confirm the diagnosis and provide a detailed description of the patient's diagnostic pathway on the basis of the electronic medical record and discharge letters from hospitals and specialists (e.g., dates of reported symptoms, encounters, tests, referrals, and involvement of other providers). GP involvement was defined on the basis of the question: 'Were you/your general practice involved in diagnosing the cancer?' (yes/no). The questionnaire also requested information about the symptoms presented by the patients at the first consultation and about the GP's interpretation of those symptoms, that is, as either alarm symptoms suggestive of cancer, symptoms suggestive of any serious illness, or vague symptoms not directly suggestive of cancer or other serious illness. This allowed us to distinguish between patients presenting with either 'alarm or any serious symptoms' or 'vague symptoms'. The GP's symptom interpretation was subjective, that is, not based on a prespecified list of alarm symptoms. The data collection has been described in further detail elsewhere (Hansen, 2008; Hansen et al, 2008).

\section{Data on covariates}

We obtained information on age and sex from the civil registry number. The patients' complete hospital discharge history, 10 years before the date of first presentation of symptoms to the GP, was used to compute a Charlson Comorbidity Index score (Charlson et al, 1987). We grouped levels of comorbidity into 'no comorbidity' (patients with no recorded disease), 'patients with moderate comorbidity' (index score of 1 and 2), and 'patients with high comorbidity' (index score of $\geqslant 3$ ). To describe data in further detail, we obtained information from the Danish National Registry of Patients on emergency admission (yes/no) and from the Danish Cancer Registry on previous cancers in other sites (yes/no) and tumour staging classified according to the TNM staging system. We re-grouped the staging information using the following principle: stage I (T1-2/N0/M0), II (T3-4/ N0/M0), III (T1-4/ N1-2/M0), IV (T1-4/N0-2/M1), and unknown.

\section{Defining time and mortality}

From the Danish Civil Registration System we retrieved information on migration and death. The study outcome was death. All patients were followed for at least 3 years after diagnosis. When comparing mortality in patients with participating and nonparticipating GPs, the date of the first-time discharge diagnosis of cancer in the County Hospital Discharge Registry was taken to be the date of diagnosis.

\section{Statistical analyses}

We stratified analyses according to the interpretation of symptoms by the GP (alarm or any serious symptoms $v s$ vague symptoms), as we expected the diagnostic pathways to be very different for these two groups of patients. We initially stratified data into colon and rectal cancer, as suggested by Ramos et al (2007), but, as the effect was constant across tumour site, data were pooled to enhance statistical precision.

The diagnostic interval was grouped into 'short' $0-4$ weeks $(0-$ 28 days), 'medium' 5-11 weeks (29-77 days), and 'long' $\geqslant 12$ 
weeks ( $78+$ days) roughly based on the 25 th and 75 th percentiles and in accordance with the waiting time guarantee of 4 weeks endorsed by the Danish Government (Olesen et al, 2009).

To estimate the odds ratio (OR) of experiencing long vs short and medium diagnostic intervals for patients presenting with vague symptoms $v s$ patients presenting with alarm or any serious symptoms, we used logistic regression and adjusted for tumour site (colon/rectal), Charlson Comorbidity Index $(0 / 1-2 / \geqslant 3)$, age $(18-59 / 60-74 / \geqslant 75)$, and sex.

Patients who died on the date of diagnosis were included in the analyses. The survival function up till 3 years after diagnosis was plotted for each of the three groups of diagnostic interval. We used logistic regression to estimate 1-year and 3-year mortality $\mathrm{OR}$ as a function of the diagnostic interval adjusted for tumour site, Charlson Comorbidity Index, age, and sex. We modelled the data in two ways: first, we calculated the adjusted OR for short or long $v s$ medium diagnostic intervals.

Second, we treated the diagnostic interval as a continuous variable using restricted cubic splines in order to make efficient use of withincategory information (Greenland, 1995). We chose an a priori reference point of 4 weeks ( 28 days) and used four knots.

To address the possibility that emergency admission was a confounder and not an intermediate step in the diagnostic pathway, we additionally adjusted for emergency admission and repeated the analyses after excluding patients with emergency admissions.

We calculated $95 \%$ confidence intervals (95\% CIs) for all estimates and tested each model against a model with no diagnostic interval term using the Wald test. A two-sided $P$-value of $\leqslant 0.05$ was defined as statistically significant.

\section{RESULTS}

We identified 363 incident CRC patients over the age of 17 years. In $58(16.0 \%)$ of the cases, the GPs did not participate or complete the questionnaire (Figure 1). We found no discrepancies between patients with participating GPs and nonparticipating GPs with respect to 1-year and 3-year mortality after discharge, age, gender, comorbidity, tumour stage, previous cancer, or emergency admission (Table 1).

Out of the 305 incident CRC patients with participating GPs, we excluded $37(12.9 \%)$ for whom the GP was not involved in diagnosing the cancer (Figure 1). Compared with the 268 patients with GP involvement, patients with uninvolved GPs were more likely to be admitted to hospital as an emergency (Table 1).

The odds of experiencing long $v s$ short diagnostic intervals (adjusted for comorbidity, age, and sex) were 3.99-fold (95\% CI: $2.10,7.56)$ higher for the 67 patients presenting with vague symptoms than for the 201 patients presenting with alarm symptoms or any serious symptoms. Patients presenting with vague symptoms had lower levels of comorbidity and were less likely to present with blood in stools than patients with clear symptoms (Table 2 ).

\section{Diagnostic interval and mortality}

Overall, $63(23.5 \%)$ CRC patients died within 1 year, and 113 $(42.2 \%)$ within 3 years of diagnosis (Figure 2 ).

In patients presenting with alarm symptoms or any serious symptoms, the adjusted 3-year mortality OR was 2.56 (95\% CI: $1.29,5.05)$ for short and $2.04(95 \% \mathrm{CI}: 0.87,4.77)$ for long compared with medium diagnostic intervals (Table 3 ). The cubic splines regression analysis (Figure 3 ) revealed that the risk of dying within 3 years began to increase after 5 weeks of diagnostic interval $(P=0.002)$.

In patients presenting with vague symptoms, the adjusted 3-year mortality OR was 0.71 (95\% CI: 0.32, 2.91) for long compared with medium diagnostic intervals. As only 10 patients had short
Table I Cumulative mortality and clinical features for the study base of 363 of incident colorectal cancer patients

\begin{tabular}{|c|c|c|c|c|}
\hline & \multicolumn{2}{|c|}{ Participating GP } & \multirow[b]{2}{*}{$\begin{array}{l}\text { Nonparti- } \\
\text { cipating } \\
\text { GP }\end{array}$} & \multirow[b]{2}{*}{$\begin{array}{l}\text { Total } \\
\text { study } \\
\text { base }\end{array}$} \\
\hline & $\begin{array}{l}\text { Involved } \\
\text { GP }\end{array}$ & $\begin{array}{c}\text { Uninvolved } \\
\text { GP }\end{array}$ & & \\
\hline$N$, patients (\%) & $268(73.8)$ & $37(10.2)$ & $58(16.0)$ & $363(100)$ \\
\hline $\begin{array}{l}\text { I-year mortality } \\
\text { after discharge }\end{array}$ & $66(24.6)$ & $13(35.1)$ & $21(36.2)$ & $100(27.5)$ \\
\hline $\begin{array}{l}\text { 3-year mortality } \\
\text { after discharge }\end{array}$ & $115(42.9)$ & $20(54.1)$ & $27(46.6)$ & $162(44.6)$ \\
\hline \multicolumn{5}{|l|}{ Tumour site } \\
\hline Colon & $183(68.3)$ & $30(81.1)$ & $4 \mid(70.7)$ & $254(70.0)$ \\
\hline Rectal & $85(31.7)$ & $7(18.9)$ & $17(29.3)$ & $109(30.0)$ \\
\hline \multicolumn{5}{|l|}{ Age at discharge } \\
\hline Median years $(|\mathrm{Q}|)$ & $70(62-80)$ & $74(67-80)$ & $72(62-80)$ & $72(63-80)$ \\
\hline $18-59$ years & $56(20.9)$ & $5(13.5)$ & $12(22.4)$ & $73(20.1)$ \\
\hline $60-74$ years & $104(38.8)$ & $15(40.5)$ & $21(34.5)$ & $140(38.6)$ \\
\hline$\geqslant 75$ years & $108(40.3)$ & $17(45.9)$ & $25(43.1)$ & $150(41.3)$ \\
\hline \multicolumn{5}{|l|}{ Gender } \\
\hline Female & $132(49.3)$ & $16(43.2)$ & $29(50.0)$ & $177(48.8)$ \\
\hline Male & $136(50.7)$ & $21(56.8)$ & $29(50.0)$ & $186(51.2)$ \\
\hline \multicolumn{5}{|c|}{ Charlson Comorbidity Index at discharge } \\
\hline Low $(0)$ & $139(51.9)$ & $17(45.9)$ & $31(53.4)$ & $187(5 \mid .5)$ \\
\hline Moderate $(1-2)$ & $97(36.2)$ & $13(35.1)$ & $18(31.0)$ & $128(35.3)$ \\
\hline High $(\geqslant 3)$ & $32(11.9)$ & $7(18.9)$ & $9(15.5)$ & $48(13.2)$ \\
\hline \multicolumn{5}{|l|}{ Previous cancer } \\
\hline Yes & $40(\mid 4.9)$ & $10(27.0)$ & $13(22.4)$ & $63(17.4)$ \\
\hline No & $228(85.1)$ & $27(73.0)$ & $45(77.6)$ & $300(82.6)$ \\
\hline \multicolumn{5}{|l|}{ Tumour stage (TNM) } \\
\hline I & $25(9.3)$ & $2(5.4)$ & $6(10.3)$ & $33(9.1)$ \\
\hline ॥ & $94(35.1)$ & $10(27.0)$ & $22(37.9)$ & $126(34.7)$ \\
\hline III & 7| (26.5) & II (29.7) & II (19.0) & $93(25.6)$ \\
\hline IV & $56(20.9)$ & $6(16.2)$ & $13(22.4)$ & 75 (20.7) \\
\hline Unknown & $22(8.2)$ & $8(21.6)$ & $6(10.3)$ & $36(9.9)$ \\
\hline \multicolumn{5}{|l|}{ Emergency admission } \\
\hline Yes & $65(24.3)$ & $21(56.8)^{\mathrm{a}}$ & $17(29.3)$ & $103(28.4)$ \\
\hline No & $203(75.7)$ & $16(43.2)$ & $4 \mid(70.7)$ & $260(71.6)$ \\
\hline
\end{tabular}

Abbreviations: $\mathrm{GP}=$ general practitioner; $\mid \mathrm{Q} \mathrm{I}=$ interquartile interval; $\mathrm{TNM}=$ tumour, node, metastasis. ${ }^{a} P \leqslant 0.05$ using a log-rank test for equality of survivor functions and a $\chi^{2}$ test for difference between groups comparing (I) patients with participating and nonparticipating GPs and (2) patients with involved and uninvolved participating GPs. No statistically significant differences were found between the former groups.

diagnostic intervals, comparison with this group was not justified. The cubic splines regression analysis (Figure 3 ) revealed a reverse effect with increasing risk of dying from day 1 and up to $\sim 12$ weeks, but the association was not statistically significant $(P=0.205)$

When analysing 1-year mortality (Table 3), adjusting for emergency admission or excluding patients with emergency admissions, we found similar trends and no major changes in estimates (data not shown).

\section{DISCUSSION}

In this population-based cohort study of 268 incident CRC patients attending primary care before diagnosis, mortality decreased with diagnostic intervals up to $\sim 5$ weeks, after which mortality increased. The counterintuitive finding of short waiting time being 
Table 2 Characteristics of 268 incident colorectal cancer patients with GP involvement presenting with either: (a) alarm symptoms of cancer or symptoms related to any serious illness or with (b) vague or ill-defined symptoms not directly related to cancer or any other serious illness

\begin{tabular}{|c|c|c|c|}
\hline & $\begin{array}{l}\text { (a) Alarm or } \\
\text { any serious } \\
\text { symptoms }\end{array}$ & $\begin{array}{l}\text { (b) Vague } \\
\text { symptoms }\end{array}$ & Total \\
\hline Number of subjects (\%) & $201(75)$ & $67(25)$ & $268(100)$ \\
\hline \multicolumn{4}{|c|}{$\begin{array}{l}\text { Diagnostic interval (time from first presentation of symptoms in primary } \\
\text { care to diagnosis)* }\end{array}$} \\
\hline Median days (IQI) & $37(21-68)$ & $74(40-152)$ & $40(23-7 \mid)$ \\
\hline \multicolumn{4}{|c|}{ Age at first presentation of symptoms in primary care } \\
\hline Median years (IQI) & $71(62-79)$ & $70(62-8 I)$ & $7 \mid(62-80)$ \\
\hline Age groups & $n(\%)$ & $\mathrm{n}(\%)$ & $\mathrm{n}(\%)$ \\
\hline $18-59$ years & $41(20)$ & $15(22)$ & $56(21)$ \\
\hline $60-74$ years & $76(38)$ & $29(43)$ & $105(39)$ \\
\hline$\geqslant 75$ years & $84(42)$ & $23(34)$ & $107(40)$ \\
\hline \multicolumn{4}{|l|}{ Sex } \\
\hline Female & $95(47)$ & $37(55)$ & $132(49)$ \\
\hline Male & $106(53)$ & $30(45)$ & $\mid 36(5 \mid)$ \\
\hline \multicolumn{4}{|c|}{ Comorbidity at first presentation of symptoms in primary care*** } \\
\hline Low $(0)$ & $115(57)$ & $52(78)$ & $167(62)$ \\
\hline Moderate $(1-2)$ & $67(33)$ & $12(18)$ & 79 (29) \\
\hline $\operatorname{High}(\geqslant 3)$ & $19(9)$ & $3(4)$ & $22(8)$ \\
\hline \multicolumn{4}{|l|}{ Previous cancer } \\
\hline Yes & $30(15)$ & $10(15)$ & $40(15)$ \\
\hline No & $17 \mid(85)$ & $57(85)$ & $228(85)$ \\
\hline \multicolumn{4}{|l|}{ Tumour stage (TNM) } \\
\hline 1 & $20(10)$ & $5(7)$ & $25(9)$ \\
\hline$\|$ & $73(36)$ & $21(31)$ & $94(35)$ \\
\hline III & $54(27)$ & $17(25)$ & $71(26)$ \\
\hline IV & $37(18)$ & $19(28)$ & $56(21)$ \\
\hline Unknown & $17(8)$ & $5(7)$ & $22(8)$ \\
\hline \multicolumn{4}{|l|}{ Emergency admission } \\
\hline Yes & $43(21)$ & $22(33)$ & $65(24)$ \\
\hline No & $158(79)$ & $45(67)$ & $203(76)$ \\
\hline \multicolumn{4}{|c|}{ Patient presented with change in bowel habits } \\
\hline Yes & $10 \mid(50)$ & $25(37)$ & $126(47)$ \\
\hline No & $10 \mid(50)$ & $42(63)$ & $143(53)$ \\
\hline \multicolumn{4}{|c|}{ Patient presented with blood in stools*** } \\
\hline Yes & $94(47)$ & $7(10)$ & 101 (38) \\
\hline No & 107 (53) & $60(90)$ & 167 (62) \\
\hline
\end{tabular}

Abbreviations: $\mathrm{GP}=$ general practitioner; $|\mathrm{Q}|=$ interquartile interval; $\mathrm{TNM}=$ tumour, node, metastasis. *P $\leqslant 0.05$ using independent sample $t$-test for difference in median diagnostic intervals (log transformed distributions) comparing alarm or any serious with vague. $* * P \leqslant 0.05$ using $\chi^{2}$ test for difference between groups (alarm or any serious vs vague symptoms)

associated with high mortality only applied to patients presenting with alarm symptoms or any serious symptoms. In the quarter of patients presenting with vague symptoms, the association was reverse although not statistically significant.

\section{Strengths of the study}

The strengths of the study lie in the population-based design made possible by a uniformly organised health-care system and validation using histological confirmation of diagnoses and complete follow-up, all of which reduced selection and information bias.
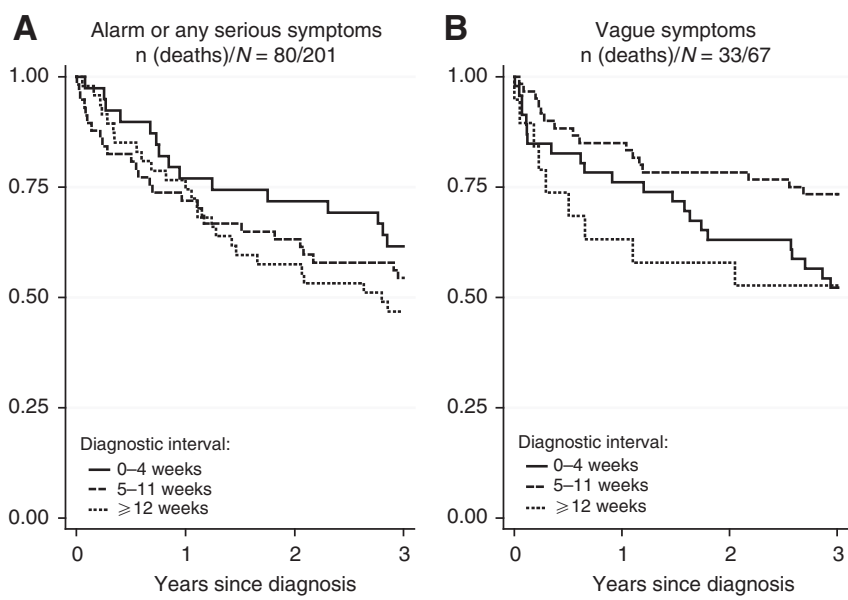

Figure 2 Estimated survival according to the length of diagnostic nterval analysed for colorectal cancer patients presenting with $(\mathbf{A})$ alarm symptoms of cancer or symptoms related to any serious illness and (B) vague or ill-defined symptoms not directly related to cancer or any other serious illness. The solid curves indicate $0-4$ weeks; dashed curves indicate $5-1 \mid$ weeks; and dotted curves indicate $\geqslant 12$ weeks from first presentation of symptoms in primary care to diagnosis (the diagnostic interval).

Table 3 Cumulative mortality (MR) and adjusted 3-year mortality ORs for 268 incident colorectal cancer patients with GP involvement, according to the length of the diagnostic interval (time from first presentation of symptoms in primary care to diagnosis).

\begin{tabular}{|c|c|c|c|c|}
\hline $\begin{array}{l}\text { Diagnostic } \\
\text { interval }\end{array}$ & $\mathbf{N}$ & MR & $\begin{array}{c}\text { Crude } \\
\text { OR }(95 \% \mathrm{Cl})\end{array}$ & $\begin{array}{c}\text { Adjusted }^{\mathrm{a}} \\
\text { OR }(95 \% \mathrm{Cl})\end{array}$ \\
\hline \multicolumn{5}{|c|}{ 3-year mortality after diagnosis } \\
\hline \multicolumn{5}{|c|}{ (a) Patients presenting with alarm or any serious symptoms $(\mathrm{N}=201)$} \\
\hline $0-4$ weeks & 75 & $48 \%$ & $2.15(1.14-4.08)$ & $2.56(1.29-5.05)$ \\
\hline $5-11$ weeks & 90 & $30 \%$ & I (ref.) & I (ref.) \\
\hline$\geqslant 12$ week & 36 & $47 \%$ & $2.09(0.94-4.62)$ & $2.04(0.87-4.77)$ \\
\hline \multicolumn{5}{|c|}{ (b) Patients presenting with vague symptoms $(\mathrm{N}=67)$} \\
\hline $0-4$ weeks & 10 & $10 \%$ & & $\begin{array}{l}\text { Comparison } \\
\text { not justified }\end{array}$ \\
\hline $5-11 n$ & 27 & $56 \%$ & $1(r$ & I (ref.) \\
\hline & 30 & $57 \%$ & $0.96(0.37-2.98)$ & $0.7 \mid(0.32-2.9 \mid)$ \\
\hline \multicolumn{5}{|c|}{ I-year mortality after diagnosis } \\
\hline \multicolumn{5}{|c|}{ (a) Patients presenting with alarm or any serious symptoms $(N=201)$} \\
\hline $0-4$ weeks & 75 & $25 \%$ & $1.70(0.79-3.63)$ & $2.09(0.92-4.73)$ \\
\hline $5-11 y$ & 90 & 179 & $1(1$ & I (ref.) \\
\hline$\geqslant 12$ weeks & 36 & $31 \%$ & $2.20(0.89-5.4 I)$ & $2.35(0.88-6.26)$ \\
\hline \multicolumn{5}{|c|}{ (b) Patients presenting with vague symptoms $(\mathrm{N}=67)$} \\
\hline $0-4$ weeks & 10 & $10 \%$ & $\begin{array}{l}\text { Comparison } \\
\text { not justified }\end{array}$ & $\begin{array}{l}\text { Comparison } \\
\text { not justified }\end{array}$ \\
\hline $5-11$ & 27 & 37 & I (ref.) & I (ref.) \\
\hline$\geqslant 12$ weeks & 30 & $23 \%$ & $0.52(0.16-1.64)$ & $0.40(0.11-1.48)$ \\
\hline
\end{tabular}

Abbreviations: $\mathrm{GP}=$ general practitioner; $\mathrm{OR}=$ odds ratio; $\mathrm{Cl}$, confidence interval. ${ }^{a}$ Adjusted for tumour site (colon/rectal), Charlson Comorbidity Index $(0 / 1-2 / \geqslant 3)$, age $(\mid 8-59 / 60-74 / \geqslant 75)$, and sex. To address the possibility that emergency admission was a confounder and not an intermediate step in the diagnostic pathway, we additionally adjusted for emergency admission and repeated the analyses after excluding patients with emergency admissions. In both subanalyses, we saw comparable trends and no changes in estimates (data not shown).

The high response rate among GPs $(84.0 \%)$ also reduced the potential for selection bias. By excluding patients in whose diagnosis the GPs had not been involved, we ensured a more accurate measure of the exposure (the diagnostic interval) and a 
A

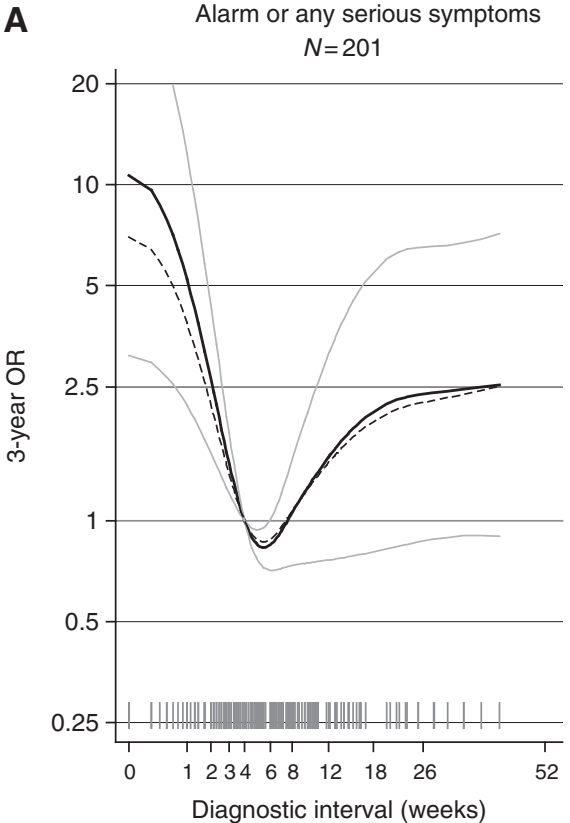

B
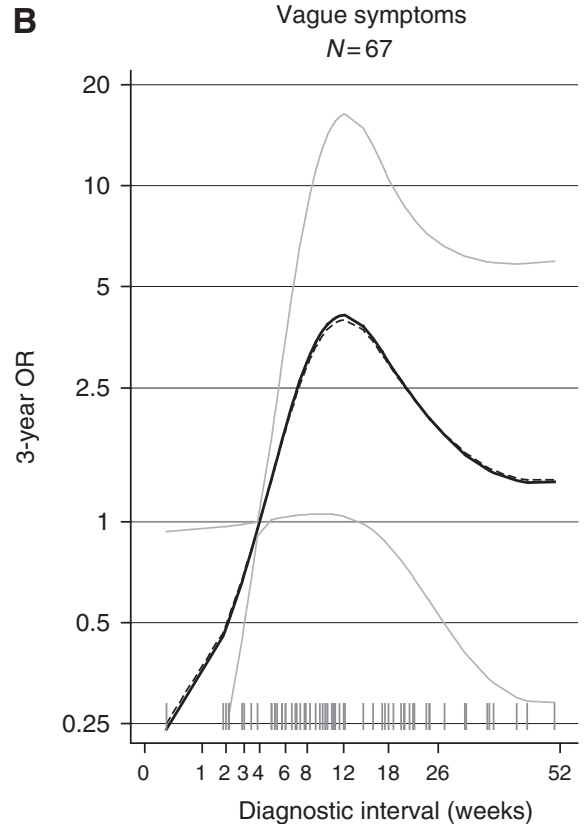

Figure 3 Estimated 3-year mortality odds ratios (ORs) as a function of the diagnostic interval (time from first presentation of symptoms in primary care until diagnosis) analysed for colorectal cancer patients presenting with (A) alarm symptoms of cancer or symptoms related to any serious illness and (B) vague or ill-defined symptoms not directly related to cancer or any other serious illness. We adjusted for tumour site (colon/rectal), Charlson Comorbidity Index ( $0 / 1-2 / \geqslant 3)$, age $(18-59 / 60-74 / \geqslant 75)$, and sex. The solid curves indicate adjusted estimates with point-wise $95 \%$ confidence limits in grey. The dashed curves indicate crude estimates. The grey spikes show the distribution of the diagnostic intervals on a squared scale. The grey horizontal lines indicate the chosen reference point of 4 weeks (28 days).

more homogeneous group with respect to confounders; and thus obtained better internal validity. Furthermore, the analyses were strengthened by allowing between-site variability in delay and mortality and a nonmonotonic effect (Maguire et al, 1994); and by addressing confounding by indication as discussed further below.

\section{Limitations of the study}

The limitations relating to the study design include possible selection bias, information bias, and residual confounding.

First of all, $16.0 \%$ of the study base could not be included in the final analyses because of nonparticipation of GPs. As seen on Table 1, patients with nonparticipating GPs shared many of the same clinical features as patients with uninvolved GPs, including equal mortality rates. Thus, the GPs may not have been involved in diagnosing cancer for a substantial part of these patients (if patients, e.g., presented with emergency conditions and bypassed their GP). Nevertheless, we cannot reject that some GPs may have chosen not to participate, because they retrospectively believed they had caused undue delays in diagnosing cancer. Given the observed convex and concave trends, it is difficult to predict the direction of the bias due to selection.

Second, given the retrospectively collected data and the comprehensive knowledge of GPs of their patients, we cannot rule out differential misclassification regarding the length of the diagnostic interval. Such misclassification would occur if, for example, the GPs tended to understate the lengths of the diagnostic intervals for patients who died shortly after diagnosis or were diagnosed with advanced-stage cancers. If this were to be the case, it would explain some of the excess mortality among patients with short diagnostic intervals. To reduce information bias, GPs were encouraged to consult their electronic medical records.

The consistent findings of higher mortality for short diagnostic intervals among patients presenting with alarm symptom or any serious symptoms - even after adjustment and exclusion of patients with emergency admissions - could also be an indication of residual confounding. Unknown confounding such as tumour aggressiveness may have reversed the association, as patients with rapidly growing tumours may exhibit more symptoms that may raise appraisal and lead to shorter diagnostic intervals, but ever worse outcomes. Although we were unable to account for the speed of tumour growth and/or aggressive malignancies, we partly dealt with confounding by appraisal by stratifying data according to the symptom interpretation of the GP. This will be discussed further below.

Finally, although it was an important tool in limiting the risk of confounding, the stratification procedure and restriction also reduced the power of the study. A larger study is needed to assess the effect in patients presenting with vague symptoms.

\section{COMPARISON WITH FINDINGS FROM OTHER STUDIES}

Most previous studies have examined the influence of total delay (time from first symptom to treatment), total diagnostic delay (time from first symptom to diagnosis), or provider delay (time from first presentation of symptoms to treatment). Common for all these studies is the mixing of patient, diagnostic, and/or treatment delays. From a methodological point of view, it is particularly tricky that both the prognostic factors and the premises for sorting patients are likely to change during the pathway to treatment. The best example is tumour stage, which is an important prognostic factor at the time of diagnosis and surely a confounder of the association between treatment delay and mortality. But, it does not qualify as a confounder of the association between diagnostic delay and mortality because it succeeds presentation. If we control for this factor in studies of diagnostic delay, we create confounding.

Over the past three decades, the association between time to diagnosis and mortality has been the subject of at least 14 studies 
based on data from symptomatic colon and/or rectal cancer patients (Irvin and Greaney, 1977; Polissar et al, 1981; Pescatori et al, 1982; Hillon et al, 1985; Goh et al, 1987; Bako et al, 1988; Porta et al, 1991; Auvinen, 1992; Ponz de Leon et al, 1992; Maguire et al, 1994; Fernandez et al, 2002; Rupassara et al, 2006; Stapley et al, 2006; Terhaar et al, 2010). The studies report either no statistically significant association or the waiting time paradox. The present study is pioneering in the sense that it challenges the premises of former models. The finding of a U-shaped association between the length of the diagnostic interval and mortality after diagnosis of cancer provides evidence that previous studies may not have represented reality very well; either because they did not allow for a continuous, nonmonotonic effect (Hillon et al, 1985; Porta et al, 1991; Rupassara et al, 2006) or because they introduced bias by adjusting for intermediate variables such as tumour stage (Polissar et al, 1981; Fernandez et al, 2002; Stapley et al, 2006; Terhaar et al, 2010). This is the first study to address these issues while at the same time controlling for confounding factors at first presentation of symptoms in primary care.

Apart from contesting previous models, we believe that the results may also challenge former reasoning. It has often been speculated that the consistent findings of higher mortality for short delays was caused by hidden confounders like the tumour's speed of growth and/or its ability to spread (Pescatori et al, 1982; Auvinen, 1992; Rupassara et al, 2006). According to this theory, aggressive tumours are associated with shorter delays because they are easier to appraise (Neal, 2009). However, for this theory to hold true, we would expect patients presenting with vague symptoms to have an overall better survival than patients presenting with alarm or any serious symptoms and to see similar trends for the two groups. Apparently, this is not the case.

\section{Underlying mechanisms and clinical implications}

We believe that the contradictory findings of the waiting time paradox mainly reflects confounding by indication, that is, a bias stemming from the inherent difference in prognosis of patients given different medical priority (Rothman, 2006). This mechanism occurs if GPs, and subsequently diagnostic imaging centres and endoscopic centres, as expected, give priority to seriously ill patients who may have higher inherent mortality (the 'sick-quick' group), whereas being more reluctant to expedite patients with less obvious symptoms of cancer (the 'low risk-slow diagnosis' group). This may even be supported by the fast-track guidelines. The association of short diagnostic intervals and high mortality in patients with alarm or serious symptoms and corresponding long diagnostic intervals and low mortality in patients with vague symptoms strongly supports the presence of this mechanism.

To conclude, most of the potential biases (from selection, misclassification, confounding, and/or random error) will either have influenced the results towards the null hypothesis or reversed the association. Based on this observational study, we can only speculate on the exact cause of the increased mortality with longer diagnostic intervals. A biological explanation could be that the exponential growth of the tumours predicates early diagnosis as a crucial factor even for patients presenting with vague symptoms. However, it should always be kept in mind that observational studies do not test the hypothesis that expediting diagnosis provides a mortality benefit. The diagnostic interval is a highly complex variable reflecting tumour biology, patient behaviour, the clinical pathway, and the functioning of the health-care system. This study's significance is that it provides some evidence against the hypothesis that the diagnostic interval has no effect on mortality and thus speaks against the tendency to downplay the importance of the diagnostic interval.

\section{CONCLUSION}

On the basis of detailed primary-care data, this study shows the waiting time paradox together with an increasing mortality with longer diagnostic intervals in CRC patients presenting with alarm or serious symptoms.

\section{ACKNOWLEDGEMENTS}

We thank the Department of Clinical Epidemiology for contributing data on comorbidity to the study. The three anonymous journal referees made very sage points, and have improved the paper. The study was supported by grants from the Danish Cancer Society, the Novo Nordic Foundation, the Danish Graduate School in Public Health Science, and Aarhus University. The sponsors were not involved in any part of the study. The study was approved by the Danish Data Protection Agency and the Danish National Board of Health. According to the Committees on Biomedical Research Ethics in the Central Denmark Region, the Act on a Biomedical Research Ethics Committee System and the Processing of Biomedical Research Projects does not apply to this project. All authors had full access to data and take full responsibility for the accuracy of the data analysis.

\section{AUTHOR CONTRIBUTIONS}

MLT was responsible for data acquisition from public and medical databases, performed the statistical analyses, and wrote the paper. She is the guarantor. MF supervised the statistical analyses and designed programmes in Stata (version 11) (Statacorp LP, College Station, TX, USA) for generating cubic splines with specific reference values (centercsplines.ado) and estimates with standard errors for linear combinations (calcest.ado) - both applied in Figure 3. RPH designed the GP and patient questionnaires and was responsible for this part of the data acquisition. $\mathrm{PV}$ and $\mathrm{FO}$ initiated the study, and PV, MF, WH, and FO provided critical revision of the intellectual content of the paper. All authors contributed to the writing of and approved the final paper.

\section{Conflict of interest}

The authors declare no conflict of interest. FO is the chairman of the Danish Cancer Society.

\section{REFERENCES}

Atkin WS, Edwards R, Kralj-Hans I, Wooldrage K, Hart AR, Northover JM, Parkin DM, Wardle J, Duffy SW, Cuzick J (2010) Once-only flexible sigmoidoscopy screening in prevention of colorectal cancer: a multicentre randomised controlled trial. Lancet 375: 1624-1633

Auvinen A (1992) Social class and colon cancer survival in Finland. Cancer 70: $402-409$

Bako G, Hill GB, Ferenczi L, Hanson J (1988) Factors influencing the survival of patients with cancer of the colon or rectum. Chronic Dis Can 9: 101-104
Barrett J, Jiwa M, Rose P, Hamilton W (2006) Pathways to the diagnosis of colorectal cancer: an observational study in three UK cities. Fam Pract 23: $15-19$

Charlson ME, Pompei P, Ales KL, MacKenzie CR (1987) A new method of classifying prognostic comorbidity in longitudinal studies: development and validation. J Chronic Dis 40: 373-383

Christiansen T (2002) Organization and financing of the Danish health care system. Health Policy 59: $107-118$ 
Fernandez E, Porta M, Malats N, Belloc J, Gallen M (2002) Symptom-todiagnosis interval and survival in cancers of the digestive tract. Dig Dis Sci 47: 2434-2440

Goh HS, Goh CR, Rauff A, Foong WC (1987) Clinico-pathological prognostic factors of large bowel cancer in Singapore: a multivariate analysis. Ann Acad Med Singapore 16: 437-440

Greenland S (1995) Dose-response and trend analysis in epidemiology: alternatives to categorical analysis. Epidemiology 6: 356-365

Hansen RP (2008) Delay in the diagnosis of cancer. PhD thesis. Månedsskrift for Praktisk Lægegerning: Kbh

Hansen RP, Olesen F, Sorensen HT, Sokolowski I, Sondergaard J (2008) Socioeconomic patient characteristics predict delay in cancer diagnosis: a Danish cohort study. BMC Health Serv Res 8: 49

Hewitson P, Glasziou P, Irwig L, Towler B, Watson E (2007) Screening for colorectal cancer using the faecal occult blood test, Hemoccult. Cochrane Database Syst Rev, (1): CD001216

Hillon P, Faivre J, Milan C, Bedenne L, Piard F, Klepping C (1985) Cancers of the rectum and colon in the Cote-d'Or department: treatment, prognosis. Gastroenterol Clin Biol 9: 704-711

Irvin TT, Greaney MG (1977) Duration of symptoms and prognosis of carcinoma of the colon and rectum. Surg Gynecol Obstet 144: 883-886

Iversen LH, Antonsen S, Laurberg S, Lautrup MD (2009) Therapeutic delay reduces survival of rectal cancer but not of colonic cancer. Br J Surg 96: $1183-1189$

Korsgaard M, Pedersen L, Sorensen HT, Laurberg S (2006) Reported symptoms, diagnostic delay and stage of colorectal cancer: a populationbased study in Denmark. Colorectal Dis 8: 688-695

Maguire A, Porta M, Malats N, Gallen M, Pinol JL, Fernandez E (1994) Cancer survival and the duration of symptoms. An analysis of possible forms of the risk function. ISDS II Project Investigators. Eur J Cancer 30A: 785-792

Mitchell E, Macdonald S, Campbell NC, Weller D, Macleod U (2008) Influences on pre-hospital delay in the diagnosis of colorectal cancer: a systematic review. $\mathrm{Br} J$ Cancer 98: 60 - 70

Neal RD (2009) Do diagnostic delays in cancer matter? Br J Cancer 101(Suppl 2): S9-S12

NORDCAN (2009) Cancer stat fact sheets: Denmark - colorectal cancer

Olesen F, Hansen RP, Vedsted P (2009) Delay in diagnosis: the experience in Denmark. Br J Cancer 101(Suppl 2): S5-S8
Pescatori M, Maria G, Beltrani B, Mattana C (1982) Site, emergency, and duration of symptoms in the prognosis of colorectal cancer. Dis Colon Rectum 25: 33-40

Polissar L, Sim D, Francis A (1981) Survival of colorectal cancer patients in relation to duration of symptoms and other prognostic factors. Dis Colon Rectum 24: 364-369

Ponz de Leon M, Sant M, Micheli A, Sacchetti C, Di Gregorio C, Fante R, Zanghieri G, Melotti G, Gatta G (1992) Clinical and pathologic prognostic indicators in colorectal cancer. A population-based study. Cancer 69: $626-635$

Porta M, Gallen M, Malats N, Planas J (1991) Influence of 'diagnostic delay' upon cancer survival: an analysis of five tumour sites. J Epidemiol Community Health 45: 225-230

Ramos M, Esteva M, Cabeza E, Campillo C, Llobera J, Aguilo A (2007) Relationship of diagnostic and therapeutic delay with survival in colorectal cancer: a review. Eur J Cancer 43: 2467-2478

Rothman KJ (2006) Epidemiology: An Introduction. Oxford University Press: New York, NY

Rupassara KS, Ponnusamy S, Withanage N, Milewski PJ (2006) A paradox explained? Patients with delayed diagnosis of symptomatic colorectal cancer have good prognosis. Colorectal Dis 8: 423-429

Sørensen HT ed. (2009) Use of Medical Databases in Clinical Epidemiology. Department of Clinical Epidemiology, Aarhus University Hospital: Aarhus

Stapley S, Peters TJ, Sharp D, Hamilton W (2006) The mortality of colorectal cancer in relation to the initial symptom at presentation to primary care and to the duration of symptoms: a cohort study using medical records. $\mathrm{Br} J$ Cancer 95: 1321 - 1325

Terhaar JS, Oort F, van dH, Coupe V, Craanen M, Meijer G, Morsink L, Visser O, van Wanrooij R, Mulder C (2010) Does delay in diagnosing colorectal cancer in symptomatic patients affect tumor stage and survival? A population-based observational study. BMC Cancer 10: 332

cc)(1) This work is licensed under the Creative Commons (c) $\mathrm{BY}$ NG SA Attribution-NonCommercial-Share Alike 3.0 Unported License. To view a copy of this license, visit http://creativecommons. org/licenses/by-nc-sa/3.0/ 